\title{
Polyaddition of Dithiol Compounds to Divinyl Compounds: The Kinetics of the Model Addition Reaction of Thiophenols to Styrenes
}

\author{
Eiichi Kobayashi,* Takatsugu Obata, Sadahito Aoshima, \\ and Junji FurukaWA \\ Department of Industrial Chemistry, Faculty of Science and Technology, \\ Science University of Tokyo, Noda, Chiba 278, Japan
}

(Received February 17, 1990)

\begin{abstract}
The addition reaction mechanism of thiophenol with styrene was studied as a model of the polyaddition of 1,4-benzenedithiol to 1,4-divinylbenzene. The addition reaction of thiophenol to styrene occurred without a side reaction and an adduct of the anti-Markownikoff's structure $\left(\phi \mathrm{SCH}_{2} \mathrm{CH}_{2} \phi\right)$ was quantitatively obtained. The kinetic study shows that the rate-determining step of the addition reaction is the chain transfer reaction between thiophenol and $\beta$-phenylthiostyryl radical. The substituent effect of the chain transfer reaction was determined on various thiophenols and styrenes. Fairly good linear correlation was obtained for the Hammett plot of $\log k_{\mathrm{tr}}$ to $\sigma^{+}$on both monomers $\left(\rho\left(k_{\mathrm{tr}}(\mathrm{St})\right)=-0.60, \rho\left(k_{\mathrm{tr}}(\mathrm{TP})\right)=+0.64\right)$. These results are discussed in detail by comparing with polyaddition.
\end{abstract}

KEY WORDS Addition Mechanism / Substituent Effect / Reaction Order / Kinetics / Styrene Derivatives / Thiophenol Derivatives / Hammett Plot / Rate-Determining Step / 1,4-Divinylbenzene / 1,4-Benzenedithiol /

The addition reactions of thiols to olefins have been studied over eighty years. ${ }^{1}$ In the absence of radical initiators, $\mathrm{H}_{2} \mathrm{~S}$ and mercaptans add to olefins by an electrophilic mechanism and the Markownikoff's rule is followed. In the presence of radical initiators such as light ${ }^{2}$ and peroxides, ${ }^{3}$ they add to olefins and acetylenes by a radical mechanism and the orientation of the adducts is the anti-Markownikoff. ${ }^{2}$ The reaction progresses by a chain mechanism ${ }^{4}$ and Sivertz et al. discuss the rates of various elementary reactions. ${ }^{5}$ However, few efforts have been made for a polyaddition of dithiols to diolefins, except for the case of hexamethylenedithiol to 1,5hexadiene to give a crystalline polyalkylenesulfide. $^{6}$

Recently, the authors studied the polyaddition of conjugated dithiol compounds to diviny $1^{7}$ or diethyny $1^{8}$ compounds. For example, 1,4-benzenedithiol (BDT) and 1,4-divinylbenzene (DVB) polymerized alternatingly without cross-linking to give the linear polymer 1 having high molecular weight $\left(\bar{M}_{w}=30000\right.$ 100000). Interestingly, the polymer has high refractive index $\left(n_{\mathrm{D}}^{18}=1.80\right)$.

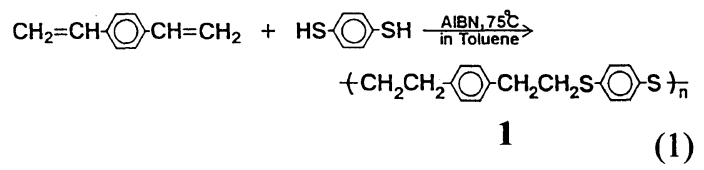

Styrene derivatives were also synthesized by the addition reaction of thiophenols to DVB in a good yield. ${ }^{9}$ The reactivity of one of the vinyl groups in DVB is about five times higher than that of the vinyl group in $p$-(2-phenylthio)ethylstyrene towards thiophenol. ${ }^{10}$

In the present paper, the reaction mechanism

\footnotetext{
* To whom all correspondence should be addressed.
} 
between thiophenols and styrenes was studied as models for the addition reaction of thiophenol to DVB and the polyaddition of BDT to DVB by means of a kinetic method. The substituent effect on the addition reaction of thiophenol derivatives to styrene derivatives was also evaluated by various Hammett's equations. In terms of the substituent effect, the transition state of the addition reaction is discussed.

\section{EXPERIMENTAL}

\section{Reagents}

$p$-Chloro, $p$-hydroxy, and $p$-( $t$-butyl)thiophenols were provided by Sumitomo Seika Chem. Co. and $p$-chlorostyrene was supplied by Hokkou Chem. Co. $p$-Methoxy and $p$-methylstyrenes were prepared according to the literatures. ${ }^{11-13}$ The other monomers were obtained commercially. All monomers were purified by vacuum distillation under nitrogen atmosphere before used. Their purities were confirmed to be over $99 \%$ by GC. Benzene as a reaction solvent was deaired to exclude oxygen by bubbling nitrogen gas for $3 \mathrm{~h}$ and then distilled before use. 2,2'-Azobisisobutyronitrile (AIBN) was used as received. Nitrogen gas was purified by pass through a glass column containing activated copper supported on kieselguhr at $200^{\circ} \mathrm{C}$, silica gel, anhydrous calcium chloride, and molecular sieve $3 \mathrm{~A}$ in this order.

\section{Addition Reaction}

In a two necked flask with a three-way stopcock and a W-cap, each benzene solution of styrenes $\left(0.3 \mathrm{moll}^{-1}\right)$, thiophenols $(0.3 \mathrm{~mol}$ $\left.1^{-1}\right)$, and AIBN $\left(3 \times 10^{-3} \mathrm{moll}^{-1}\right)$ was prepared using a syphon method under nitrogen atmosphere. Then, the reaction was carried out by mixing each solution by the syphon method in a reaction vessel. The reaction temperature was maintained mainly at $50^{\circ} \mathrm{C}$. The course of the reaction was monitored every $c a .10 \mathrm{~min}$ by measurement of the adduct concentrations by GC using of diphenylsulfide as the external reference.

\section{Measurements}

Gas chromatograms were made on a Shimadzu GC-4BMPF with a silicon GE SE-30 column $(2 \mathrm{~m})$ at $250^{\circ} \mathrm{C}$ using nitrogen carrier gas. Gel permeation chromatograms (GPC) were measured in chloroform on a Tosoh CCPD dual pump chromatograph equipped with polystyrene gel columns and ultraviolet/refractive index dual-mode detectors. The column was constructed by a series of Tosoh Tsk gel G2000HXL, G3000HXL, and G4000HXL. ${ }^{1} \mathrm{H}$ NMR spectra were obtained with a JEOL PMX-60si spectrometer in $\mathrm{CDCl}_{3}$ with tetramethylsilane as the internal standard. IR spectra were recorded on a Hitachi 260-50 spectrophotometer by a liquid film method. GC and FD mass spectra were obtained on a JEOL JMS-G3000 and JEOL JMS-MX110, respectively. Refractive indices were measured with an Atago Abbe refractometer 301. Sulfur contents of the adducts were determined by the modified Schoeniger method. ${ }^{14}$

\section{RESULTS AND DISCUSSION}

\section{Addition Reaction of Thiophenol to Styrene Influence of Oxygen}

The influence of oxygen on the reaction rate was investigated by the addition of dry air in the reaction vessel. A mixture of reagent solutions was prepared by a semi-closed method by using a stainless pipe syphon under purified nitrogen atmosphere to keep a constant concentration of oxygen remaining in the reaction vessel. By this procedure, the reproducibility of the reaction rate was fairly good at added oxygen being zero. A trace amount of oxygen remaining in the reaction vessel affects markedly the reaction rate as shown in Figure 1. The addition of a trace amount of dry air $(<0.02 \mathrm{ml})$ in the reaction vessel increases the reaction rate, whereas a large amount of dry air added $(\geq 0.02 \mathrm{ml})$ 


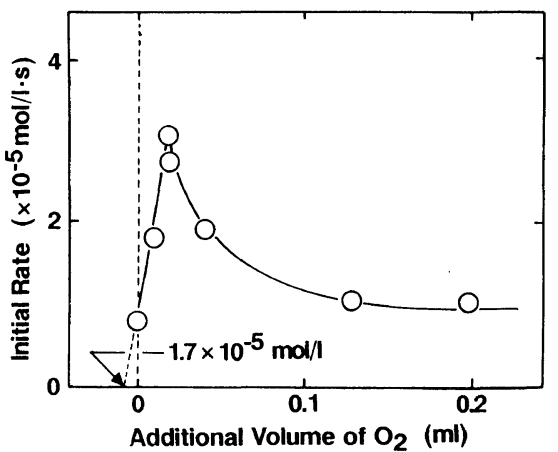

Figure 1. Effect of oxygen on the initial addition rate of thiophenol to styrene without AIBN at $40^{\circ} \mathrm{C}$ in the dark under nitrogen atmosphere. $[\phi \mathrm{SH}]_{0}=\left[\phi \mathrm{CH}=\mathrm{CH}_{2}\right]_{0}=$ $0.3 \mathrm{moll}^{-1}$ in benzene; $0.1 \mathrm{ml}$ Oxygen corresponds to $2 \times 10^{-4} \mathrm{moll}^{-1}$ in nitrogen atmosphere.

decreases the reaction rate. By extrapolation of the left hand linear part in Figure 1, oxygen remaining in the reaction system is estimated to be ca. $1.7 \times 10^{-5} \mathrm{moll}^{-1}$. When a $1 / 100$ molar quantity of AIBN to the monomers was added in the reagent mixture, the reaction rate became three hundred times faster. Therefore, the influence of oxygen remaining in the system may be negligible in the presence of AIBN.

Preparation of Addition Product of Thiophenol and Styrene

Thiophenol was reacted with an equimolar amount of styrene with AIBN initiator in benzene at $70^{\circ} \mathrm{C}$ under nitrogen atmosphere. The addition reaction proceeded without an induction period up to $100 \%$ conversion for $2 \mathrm{~h}$. The crude product was obtained by removal of the benzene from the reaction mixture without any pretreatment. More than $99 \%$ of the product was the $1: 1$ adduct of thiophenol to styrene, confirmed by GPC and GC measurements. The adduct isolated by vacuum distillation was a colorless transparent liquid $\left[\mathrm{bp} 105^{\circ} \mathrm{C} / 0.1 \mathrm{mmHg}\right.$ (lit. ${ }^{15}$ $\left.188-189^{\circ} \mathrm{C} / 15 \mathrm{mmHg}\right), n_{\mathrm{D}}^{25}=1.6060, S \mathrm{wt} \%=$ $15.1 \%$ (calcd 15.0\%), $\mathrm{m} / \mathrm{z}$ (molecular ion peak $)=214($ calcd 214$)]$. The crude product contained $c a .0 .3 \mathrm{wt} \%$ of oligomer of which molecular weight was 320 (Figure 2). Byproducts such as teromers of styrene with

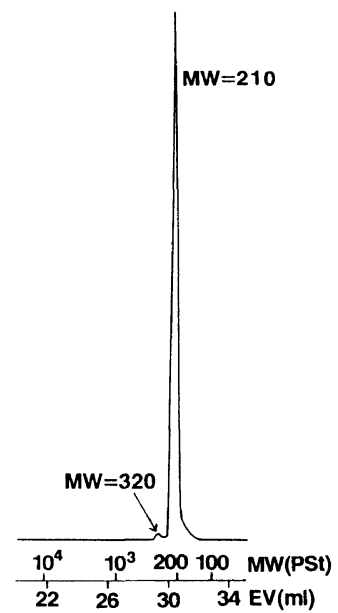

Figure 2. MWD curve of products obtained by the reaction of thiophenol to styrene at $70^{\circ} \mathrm{C}$ for $2 \mathrm{~h}$ under nitrogen atmosphere. $[\phi \mathrm{SH}]_{0}=\left[\phi \mathrm{CH}=\mathrm{CH}_{2}\right]_{0}=1 \mathrm{~mol}$ $1^{-1} ;[\mathrm{AIBN}]_{0}=1 \times 10^{-2} \mathrm{moll}^{-1}$ in benzene.

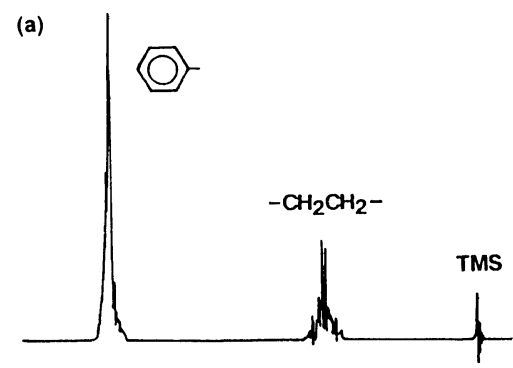

(b)

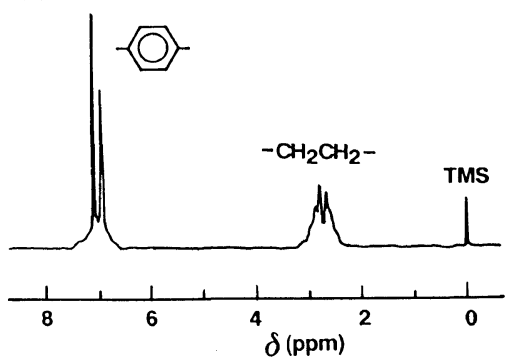

Figure 3. ${ }^{1} \mathrm{H}$ NMR spectra of (a) the adduct of thiophenol to styrene and of (b) the polymer of BDT to $\mathrm{DVB}^{7}$ in $\mathrm{CDCl}_{3}$ (JEOL JMS-FX90Q).

higher molecular weight and low boiling point materials such as diphenyldisulfides formed by the coupling reaction were not confirmed.

The structures of these products were 

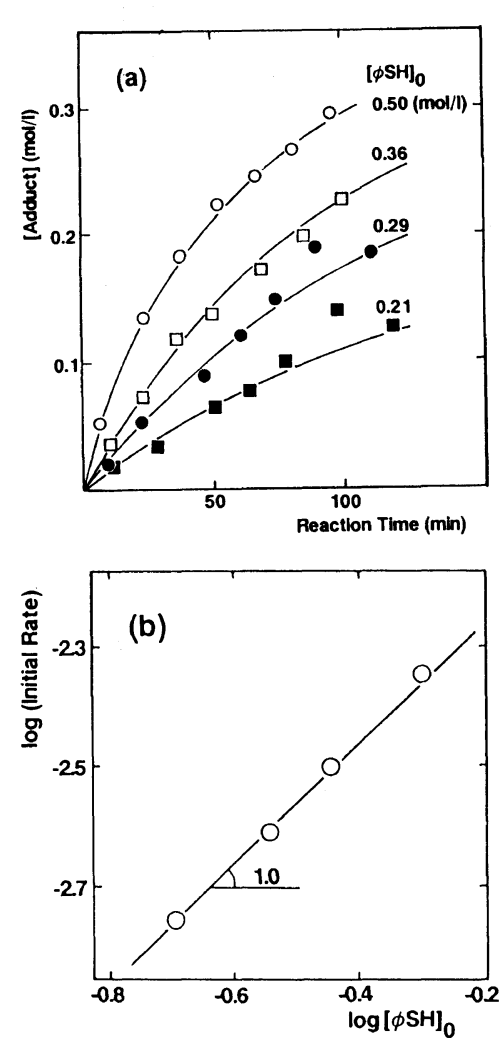

Figure 4. Time-conversion curves (a) of the adduct formation of thiophenol and styrene and reaction order (b) of thiophenol at $50^{\circ} \mathrm{C}$ in the dark under nitrogen atmosphere. $\left[\phi \mathrm{CH}=\mathrm{CH}_{2}\right]_{0}=0.28 \mathrm{moll}^{-1} ;[\mathrm{AIBN}]_{0}=$ $5.8 \times 10^{-3} \mathrm{moll}^{-1}$ in benzene. external ref. for $\mathrm{GC}$, diphenylsulfide.

estimated by ${ }^{1} \mathrm{H}$ NMR and FD mass. The ${ }^{1} \mathrm{H}$ NMR spectrum of the $1: 1$ adduct isolated by vacuum distillation shows absorptions at $\delta$ $2.85 \mathrm{ppm}$ due to methylene protons and at $\delta$ $6.50-7.60 \mathrm{ppm}$ due to benzene ring protons. Methyl and methine protons were not observed. The spectrum indicates the antiMarkownikoff's structure of the adduct. This spectrum resembles that of the polymer of BDT to DVB (Figure 3). The oligomer is estimated to be an adduct of thiophenol to styrene dimer, i.e., $\phi \mathrm{SCH}_{2} \mathrm{CH} \phi \mathrm{CH}_{2} \mathrm{CH}_{2} \phi$, because the molecular weight of it was found to be 318 from the molecular ion peak in the FD mass spectrum.

It was indicated that the addition reaction of the anti-Markownikoff's type preferentially

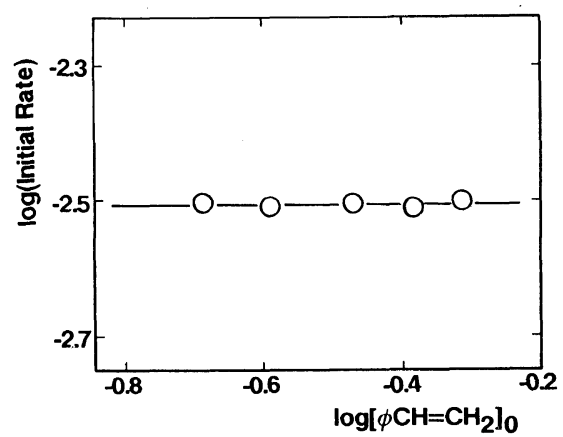

Figure 5. Reaction order of styrene at $50^{\circ} \mathrm{C}$ in the dark under nitrogen atmosphere. $[\phi \mathrm{SH}]_{0}=0.28 \mathrm{moll}^{-1}$; $\left[\phi \mathrm{CH}=\mathrm{CH}_{2}\right]_{0}=0.21-0.49 \mathrm{moll}^{-1} ; \quad[\mathrm{AIBN}]_{0}=2.9 \times$ $10^{-3} \mathrm{moll}^{-1}$ in benzene; external ref. for GC, diphenylsulfide.

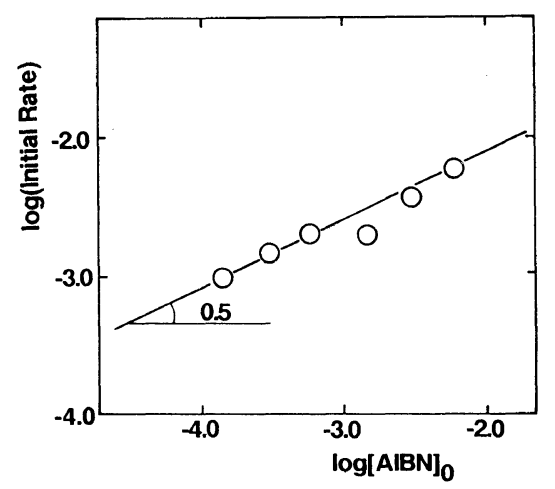

Figure 6. Reaction order of AIBN at $50^{\circ} \mathrm{C}$ in the dark under nitrogen atmosphere. $[\phi \mathrm{SH}]_{0}=0.28 \mathrm{moll}^{-1} ;[\phi \mathrm{CH}=$ $\left.\mathrm{CH}_{2}\right]_{0}=0.30 \mathrm{moll}^{-1} ; \quad[\mathrm{AIBN}]_{0}=1.5-60 \times 10^{-4} \mathrm{moll}^{-1}$ in benzene; external ref. for GC, diphenylsulfide.

occurred without appreciable side reactions under the reaction conditions. Consequently, this reaction can be utilized for the model reaction of the polyaddition of BDT to DVB.

Effects of Thiophenol, Styrene, and AIBN Concentrations on Reaction Rates

Typical time-conversion curves and reaction order thus obtained are illustrated in Figures 4 , 5, and 6. The yield of the adduct of thiophenol and styrene increases with reaction time and increasing thiophenol concentration (Figure 4 (a)). Each experimental point lays on a straight line drawn by the pseudo first order assumption. The initial rates thus obtained are plotted against thiophenol concentration (Fig- 
ure $4(\mathrm{~b}))$. The slope constant is about 1.0 indicating first order kinetics with respect to thiophenol concentration. However, the rate of adduct formation is not affected by styrene concentration (Figure 5). The kinetic order of AIBN is illustrated in Figure 6. The slope constant is approximately 0.5 . Consequently, this reaction can be expressed as the first order of thiophenol, 0.5 order of AIBN and independent on styrene concentration, i.e., the rate equation is as follows,

$$
\text { Rate }=k[\phi \mathrm{SH}]^{1}\left[\phi \mathrm{CH}=\mathrm{CH}_{2}\right]^{0}[\mathrm{AIBN}]^{0.5}
$$

where $k$ is the reaction rate constant in benzene at $50^{\circ} \mathrm{C}$.

\section{Reaction Mechanism and Rate-Determining Step}

This reaction is known to take place through a chain mechanism. ${ }^{5}$ The elementary reactions are discussed by Pallen and Sivertz ${ }^{5}$ and shown in eq $3-7$,

Initiation Reaction

$$
\begin{array}{r}
\operatorname{AIBN} \stackrel{k_{\mathrm{d}}}{\longrightarrow} 2 \dot{\mathrm{R}}+\mathrm{N}_{2} \\
\dot{\mathrm{R}}+\phi \mathrm{SH} \stackrel{k_{\mathrm{i}}}{\longrightarrow} \phi \dot{\mathrm{S}}+\mathrm{RH}
\end{array}
$$

Addition Reaction

$$
\phi \dot{\mathrm{S}}+\phi \mathrm{CH}=\mathrm{CH}_{2} \stackrel{k_{\mathrm{a}}}{\longrightarrow} \phi \underset{\mathrm{SCH}_{2}}{\dot{\mathrm{C}} \mathrm{H} \phi}
$$

Chain Transfer Reaction

$$
\dot{\mathrm{C}}+\phi \mathrm{SH} \stackrel{k_{\mathrm{tr}}}{\longrightarrow} \phi \mathrm{SCH}_{2} \mathrm{CH}_{2} \phi+\phi \dot{\mathrm{S}}
$$

\section{A}

Polymerization Reaction

$$
\dot{\mathbf{C}}+\phi \mathrm{CH}=\mathrm{CH}_{2} \stackrel{k_{\mathrm{p}}}{\longrightarrow} \dot{\mathrm{P}}
$$

Termination Reaction

$$
2 \phi \dot{\mathrm{S}} \stackrel{k_{4}}{\longrightarrow} \mathrm{T}_{4}
$$

$$
\begin{array}{r}
\dot{\mathbf{C}}+\phi \dot{\mathrm{S}} \stackrel{k_{5}}{\longrightarrow} \mathrm{T}_{5} \\
2 \dot{\mathbf{C}} \stackrel{k_{6}}{\longrightarrow} \mathrm{T}_{6}
\end{array}
$$

where $k_{\mathrm{d}}, k_{\mathrm{i}}, \cdots, k_{6}$ are reaction rate constants on each elementary reaction.

The rate equation under a steady state assumption is given as,

$$
\frac{\mathrm{d}[\mathrm{A}]}{\mathrm{d} t}=\frac{\left(\frac{k_{\mathrm{d}}[\mathrm{AIBN}]}{k_{6}}\right)^{0.5} k_{\mathrm{tr}}[\phi \mathrm{SH}]}{\left[1+\left(\frac{k_{6}}{k_{4}}\right)^{0.5} \frac{k_{\mathrm{tr}}[\phi \mathrm{SH}]}{k_{\mathrm{a}}\left[\phi \mathrm{CH}=\mathrm{CH}_{2}\right]}\right]}
$$

where $\mathrm{d}[\mathrm{A}] / \mathrm{d} t$ is the rate of the adduct formation. When the rate of the chain transfer reaction $\left(k_{\mathrm{tr}}\right)$ is extremely slower than that of the addition reaction $\left(k_{\mathrm{a}}\right)$, eq 8 is simplified as eq 9 ,

$$
\mathrm{d}[\mathrm{A}] / \mathrm{d} t=k[\phi \mathrm{SH}][\mathrm{AIBN}]^{0.5}
$$

where $k$ is $\left(k_{\mathrm{d}} / k_{6}\right)^{0.5} k_{\mathrm{tr}}$. The experimental results expressed in eq 2 agree with eq 9 . Consequently, the rate-determining step is the chain transfer reaction (eq 5).

\section{Substitutent Effect}

\section{Addition Reaction}

The addition reactions of thiophenol to styrene derivatives such as $p$-methoxy, $p$ methyl, and $p$-chlorostyrenes were carried out at $70^{\circ} \mathrm{C}$ in benzene for $2 \mathrm{~h}$ under nitrogen atmosphere. Also, instead of thiophenol, thiophenol derivatives such as $p$-methoxy, $p$-methyl, $p$-chloro, $p$-hydroxy, $p$-( $t$-butyl $)$, and $m$-methylthiophenols were used. In any monomer combination, the addition reactions occurred rapidly and were completed within $2 \mathrm{~h}$. All products obtained were transparent liquids. After fractional distillation under high vacuo, the adducts were isolated in $82-98 \%$ yields as shown in Table I. Table I summarizes the properties, IR, and ${ }^{1} \mathrm{H}$ NMR spectral data of the adducts. The structures of the adducts were confirmed to be 1-phenyl-2-phenylthio- 


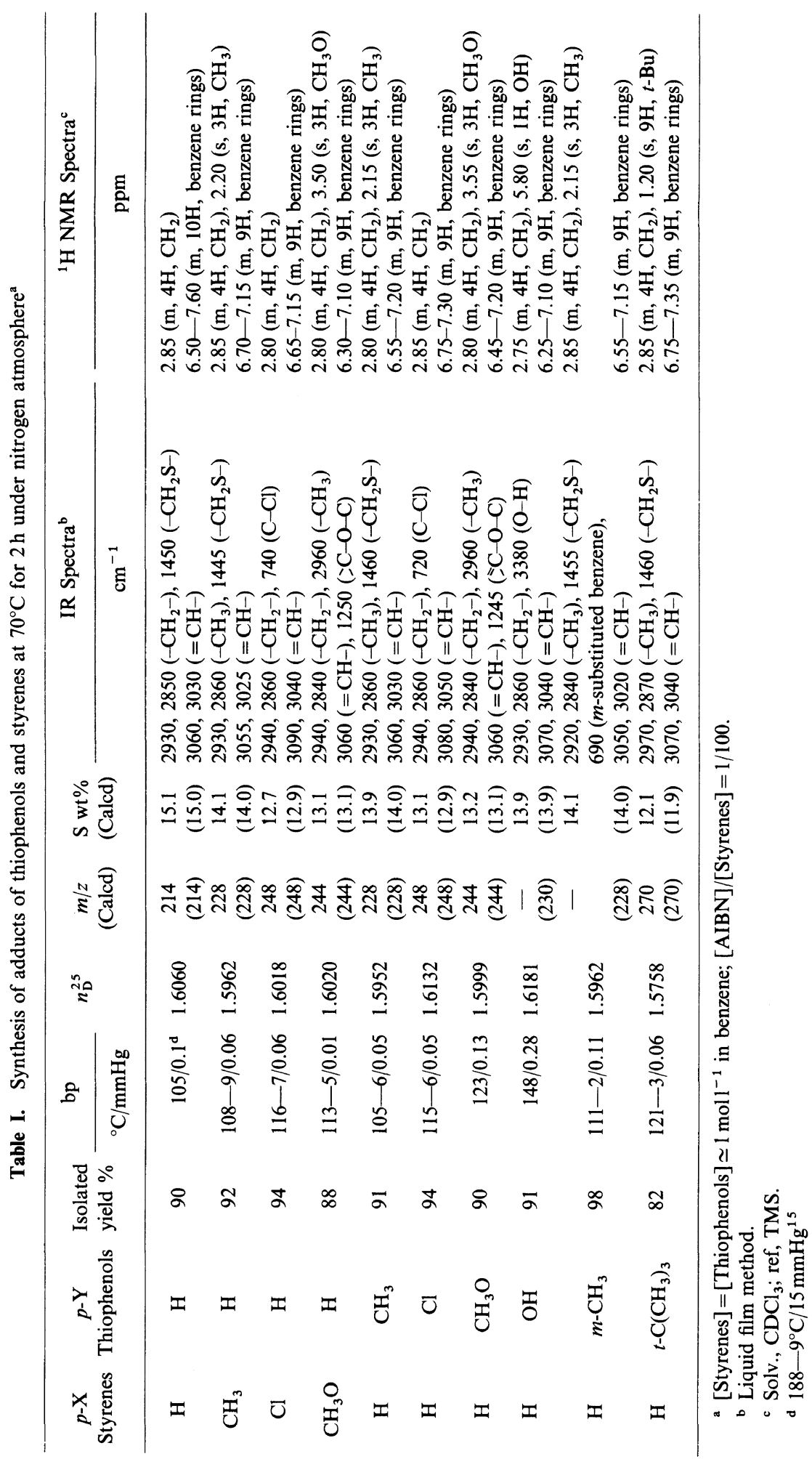


ethane derivatives by ${ }^{1} \mathrm{H}$ NMR analysis. Consequently, thiophenol derivatives added quantitatively to styrene derivatives by the anti-Markownikoff's type and side reactions were hardly occurred.

\section{Relative Rates of Adduct Formation}

By the competitive reaction of thiophenol with styrene and its derivatives the relative rate of the addition step of benzenethiyl radical to styrenes can be determined. However, the relative rate does not give information with the rate-determining step. In this study the reaction rate of the adduct formation of styrene with thiophenol or styrene derivatives with thiophenol is determined by first order plots. The relative rates are calculated to be $R^{\mathrm{X}} / R^{\mathrm{H}}$, where $R^{\mathrm{H}}$ and $R^{\mathrm{X}}$ mean the reaction rates of the adduct formation of thiophenol with styrene and $\mathrm{X}$ substituted styrenes, respectively. Here, $R^{\mathrm{X}} / R^{\mathrm{H}}$ is a function expressed by eq 10 .

$$
\begin{aligned}
\frac{R^{\mathrm{X}}}{R^{\mathrm{H}}}= & \left(\frac{k_{\mathrm{d}}[\mathrm{AIBN}]}{k_{6}^{\mathrm{X}}}\right)^{0.5} k_{\mathrm{tr}}^{\mathrm{X}} /\left(\frac{k_{\mathrm{d}}[\mathrm{AIBN}]}{k_{6}^{\mathrm{H}}}\right)^{0.5} k_{\mathrm{tr}}^{\mathrm{H}} \\
= & \frac{k_{\mathrm{tr}}^{\mathrm{X}}}{k_{\mathrm{tr}}^{\mathrm{H}}}\left(\frac{k_{6}^{\mathrm{X}}}{k_{6}^{\mathrm{H}}}\right)^{0.5}
\end{aligned}
$$

The relative rates of the chain transfer reaction, $k_{\mathrm{tr}}^{\mathbf{X}} / k_{\mathrm{tr}}^{\mathbf{H}}$ is calculated on the assumption that termination rate constants $k_{6}^{\mathbf{X}}$ and $k_{6}^{\mathbf{H}}$ of this system equal those of substituted polystyryl radicals, ${ }^{16}$ since the intermediate carbon radicals in this addition reaction are almost similar to those in the polymerization of styrenes. In the case of the adduct formation of thiophenol derivatives such as $p$-chloro, $p$-methyl, $p$-methoxy, and $p$-hydroxythiophenols to styrene, $R^{\mathrm{Y}} / R^{\mathrm{H}}$ is also similarly evaluated, where $R^{\mathrm{Y}}$ means the rate of the chain transfer reaction between $\beta$-phenylthiostyryl radical and $\mathrm{Y}$ substituted thiophenols. In this case, one is able to assume that $k_{6}^{Y}$ is equal to $k_{6}^{\mathrm{H}}$, since the effect of the substituent $\mathrm{Y}$ of $\mathrm{Y}-\phi \mathrm{SCH}_{2} \dot{\mathrm{C}} \mathrm{H} \phi$ on a reactivity of the intermediate carbon radical decreases by the methylene group. That is, $R^{\mathrm{Y}} / R^{\mathrm{H}}$ is nearly equal to $k_{\mathrm{tr}}^{\mathbf{Y}} / k_{\mathrm{tr}}^{\mathbf{H}}$.
Table II. Relative rates $\left(R^{\mathrm{X}} / R^{\mathrm{H}}\right)$ of the adduct formation and $\left(k_{\mathrm{tr}}^{\mathbf{X}} / k_{\mathrm{tr}}^{\mathrm{H}}\right)$ of the chain transfer reaction in the addition reactions of thiophenol to styrenes at $50^{\circ} \mathrm{C}$ in the dark under nitrogen atomsphere ${ }^{\mathrm{a}}$

\begin{tabular}{cccc}
\hline $\begin{array}{c}p-\mathrm{X} \\
\text { Styrenes }\end{array}$ & $\boldsymbol{R}^{\mathrm{X}} / R^{\mathrm{H}}$ & $k_{\mathrm{tr}}^{\mathbf{X}} / k_{\mathrm{tr}}^{\mathrm{H}}$ & $k_{6}^{\mathbf{X}} / k_{6}^{\mathrm{H} 16}$ \\
\hline $\mathrm{MeO}$ & 1.86 & 3.38 & 0.31 \\
$\mathrm{Me}$ & 1.57 & 2.01 & 0.61 \\
$\mathrm{Cl}$ & 0.92 & 1.10 & 0.71 \\
\hline
\end{tabular}

${ }^{\mathrm{a}}\left[\mathrm{X}-\phi \mathrm{CH}=\mathrm{CH}_{2}\right]_{0}=\left[\phi \mathrm{CH}=\mathrm{CH}_{2}\right]_{0}=[\phi \mathrm{SH}]_{0}=$ $0.30 \mathrm{moll}^{-1}$ in benzene; $[\mathrm{AIBN}]_{0} /[\phi \mathrm{SH}]_{0}=1 / 100$.

Table III. Relative rates $\left(k_{\mathrm{tr}}^{\mathbf{Y}} / k_{\mathrm{tr}}^{\mathrm{H}}\right)$ of the chain transfer reaction in the addition reactions of thiophenols to styrene at $50^{\circ} \mathrm{C}$ in the dark under nitrogen atmosphere ${ }^{a}$

\begin{tabular}{cc}
\hline $\begin{array}{c}p-\mathrm{Y} \\
\text { Thiophenols }\end{array}$ & $k_{\mathrm{tr}}^{\mathrm{Y}} / k_{\mathrm{tr}}^{\mathrm{H}}$ \\
$\mathrm{OH}$ & 0.24 \\
$\mathrm{MeO}$ & 0.31 \\
$\mathrm{Me}$ & 0.76 \\
$\mathrm{Cl}$ & 1.58 \\
\hline${ }^{\mathrm{a}}[\mathrm{Y}-\phi \mathrm{SH}]_{0}=[\phi \mathrm{SH}]_{0}=\left[\phi \mathrm{CH}=\mathrm{CH}_{2}\right]_{0}=0.3$ \\
$\mathrm{moll}^{-1}$ in benzene; $[\mathrm{AIBN}]_{0} /[\phi \mathrm{SH}]_{0}=1 / 100$.
\end{tabular}

The relative rates, $R^{\mathrm{X}} / R^{\mathrm{H}}, k_{\mathrm{tr}}^{\mathrm{X}} / k_{\mathrm{tr}}^{\mathrm{H}}$, and $k_{\mathrm{tr}}^{\mathrm{Y}} / k_{\mathrm{tr}}^{\mathrm{H}}$, are shown in Tables II and III. $K_{\mathrm{tr}}^{\mathbf{X}} / k_{\mathrm{tr}}^{\mathrm{H}}$ are calculated based on eq 10 using the experimental data of $R^{\mathrm{X}} / R^{\mathrm{H}}$ and $k_{6}^{\mathrm{X}} / k_{6}^{\mathrm{H}},{ }^{16}$ and $k_{\mathrm{tr}}^{\mathrm{Y}} / k_{\mathrm{tr}}^{\mathrm{H}}$ are used the experimental data, $R^{\mathrm{Y}} / R^{\mathrm{H}}$. In the case of styrenes, $k_{\mathrm{tr}}^{\mathbf{X}} / k_{\mathrm{tr}}^{\mathbf{H}}$ decreased in the order of $\mathrm{MeO}, \mathrm{Me}$, and then $\mathrm{Cl} . k_{\mathrm{tr}}^{\mathbf{Y}} / k_{\mathrm{tr}}^{\mathrm{H}}$ of thiophenols increased in the order of $\mathrm{OH}, \mathrm{MeO}, \mathrm{Me}$, and Cl. For example, methoxystyrene has the highest reactivity in the styrene derivatives while methoxythiophenol exhibits the lowest reactivity except hydroxythiophenol. That is, the substitutent effect of styrene derivatives is found to be nearly opposite to that of thiophenol derivatives.

The substituent group (X) on styrenes participates in the resonance stability of the intermediate carbon radical (Y- $\left.\phi \mathrm{SCH}_{2} \dot{\mathrm{C}} \mathrm{H} \phi-\mathrm{X}\right)$, and the substituent group (Y) on thiophenol contributes to the $\mathrm{S}-\mathrm{H}$ bond dissociation in the 
transition state. Accordingly, the electrondonating group on styrene derivatives destabilizes the intermediate carbon radical arising from increase in the electron density of the radical center, so that the reaction rate increases. But with thiophenol derivatives, the electron-donating group on thiophenols stabilizes the $\mathrm{S}-\mathrm{H}$ bond, so that the reaction rate decreases with increasing electron-donating power of the substituent.

These kinetic data are plotted by the Hammett's equation 11, Brown-Okamoto's equation $12,{ }^{17}$ and Yamamoto's equation $13 .{ }^{18}$
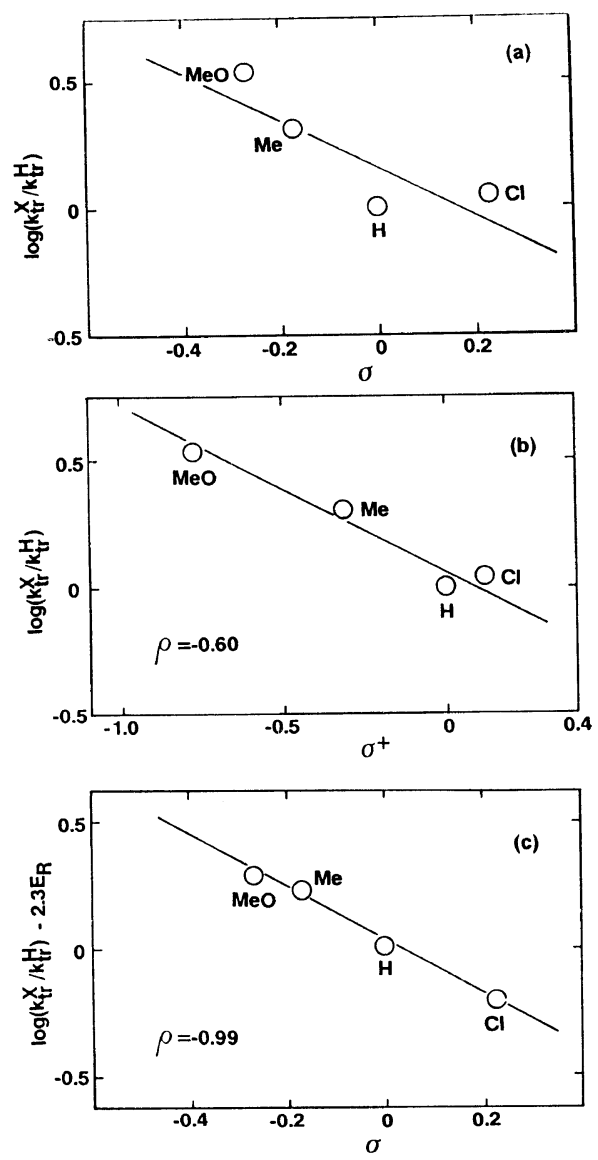

Figure 7. Hammett plots of $\log k_{\mathrm{tr}}^{\mathbf{X}} / k_{\mathrm{tr}}^{\mathrm{H}}$ of styrene derivatives at $50^{\circ} \mathrm{C}$ in the dark under nitrogen atmosphere with (a) eq 11 , (b) eq $12^{17}$ and (c) eq $13 .^{18}[\mathrm{X}$ $\left.\phi \mathrm{CH}=\mathrm{CH}_{2}\right]_{0}=\left[\phi \mathrm{CH}=\mathrm{CH}_{2}\right]_{0}=[\phi \mathrm{SH}]_{0}=0.3 \mathrm{moll}^{-1}$ in benzene; $[\mathrm{AIBN}]_{0} /[\phi \mathrm{SH}]_{0}=1 / 100$.

$$
\begin{aligned}
& \log \left(k / k_{0}\right)=\rho \sigma \\
& \log \left(k / k_{0}\right)=\rho \sigma^{+} \\
& \log \left(k / k_{0}\right)=\rho \sigma+\gamma E_{\mathbf{R}}
\end{aligned}
$$

In eq $13, \sigma$ and $E_{\mathrm{R}}$ are polar and resonance substituent constants, $\rho$ and $\gamma$ are reaction constants giving the susceptibility of the polar and resonance effects caused by the substituent, respectively. That is, $\rho \sigma$ shows a polar term and $\gamma E_{\mathbf{R}}$ is a resonance term. Figures 7 (a), (b), and (c) show Hammett plots of hydrogen abstraction from thiophenol by substituted
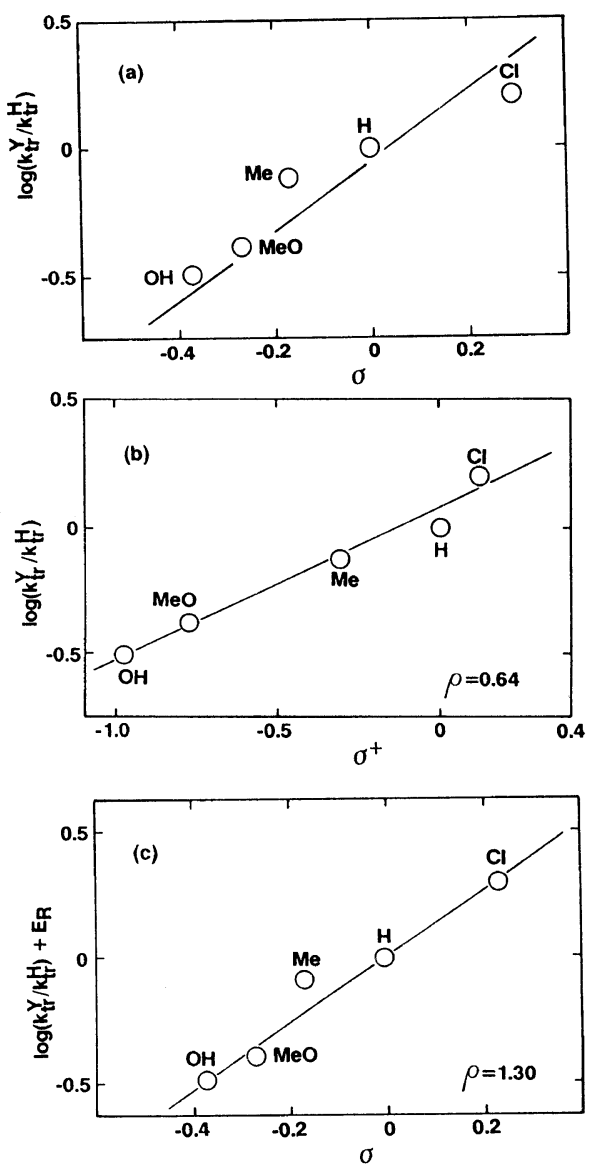

Figure 8. Hammett plots of $k_{\mathrm{tr}}^{\mathrm{Y}} / k_{\mathrm{tr}}^{\mathrm{H}}$ of thiophenol derivatives at $50^{\circ} \mathrm{C}$ in the dark under nitrogen atmosphere with (a) eq 11 , (b) eq $12^{17}$ and (c) eq $13 .^{18}[\mathrm{Y}-\phi \mathrm{SH}]_{0}=$ $[\phi \mathrm{SH}]_{0}=\left[\phi \mathrm{CH}=\mathrm{CH}_{2}\right]_{0}=0.30 \mathrm{moll}^{-1}$ in benzene; $[\mathrm{AIBN}]_{0} /[\phi \mathrm{SH}]_{0}=1 / 100$. 
Table IV. Hammett parameters obtained from Figures 7 and 8 for the addition reaction of thiophenols to styrenes

\begin{tabular}{|c|c|c|c|c|c|c|}
\hline \multirow{2}{*}{$\begin{array}{l}\text { Hammett's equation } \\
\text { No. }\end{array}$} & \multicolumn{3}{|c|}{ Substituted styrenes (X) } & \multicolumn{3}{|c|}{ Substituted thiophenols $(\mathrm{Y})$} \\
\hline & $\rho$ & $\gamma$ & $r^{\mathbf{a}}$ & $\rho$ & $\gamma$ & $r^{\mathbf{a}}$ \\
\hline$\rho \sigma$ & -0.98 & - & 0.86 & 1.40 & - & 0.95 \\
\hline $12 \rho \sigma^{+}$ & -0.60 & - & 0.98 & 0.64 & - & 0.99 \\
\hline $13 \rho \sigma+\gamma E_{\mathbf{R}}$ & -0.99 & 2.3 & 0.99 & 1.30 & -1.0 & 0.97 \\
\hline
\end{tabular}

${ }^{\text {a }}$ Correlation coefficient.

$\beta$-phenylthiostyryl radicals with eq 11,12 , and 13, respectively. Similarly, Figure 8 shows Hammett plots of hydrogen abstraction from substituted thiophenols by $\beta$-phenylthiostyryl radical. The reaction constant, $\rho$, and resonance reaction constant, $\gamma$, obtained from Figures 7 and 8 are shown in Table IV together with the correlation coefficient, $r$, calculated by least squares treatment of the experimental data. Better correlation was obtained with eq 12 and 13 rather than eq 11 because the correlation coefficient, $r$, from eq 12 and 13 is nearly one. Consequently, it is noticed that the rates of these reactions are controlled not only by the polar term but also by the resonance term.

Schaafsma et al. ${ }^{19}$ determined relative reactivities of substituted thiophenols with respect to hydrogen abstraction by 1-cyano-1cyclohexyl radical. Fairly good correlation was obtained by Hammett plots of the relative reactivity against $\sigma^{+}$. It was also found by Imoto et al. $^{20}$ that the relative reactivity of the addition reaction between substituted polystyryl radicals and styrene obeyed the simple Hammett's equation 11 while that of the addition reaction between polystyryl radical and substituted styrenes did not but eq 13. In the case of the chain transfer reaction, the substituent effect in the reaction between various polystyryl radicals and cumen derivatives obeyed eq 11 from the point of the radical side and eq 13 from the point of the cumen side. $^{21}$ Imoto $^{22}$ discuss the contribution of the intermediate structure 2 where the electron transfer from polystyryl radical to cumens in a transition state is assumed, since the reaction constant between polystyryl radical and substituted cumens was positive $(\rho=+0.7)$.

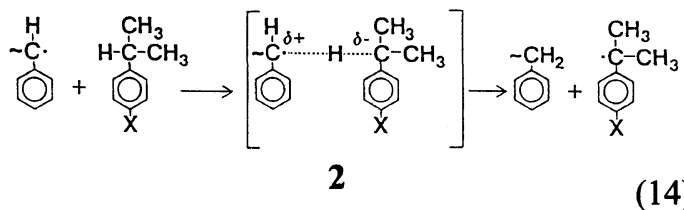

In the present study, the reaction constants, $\rho$, between $\beta$-phenylthiostyryl radical and substituted thiophenols are positive values and those between substituted $\beta$-phenylthiostyryl radicals and thiophenol are negative values as shown in Table IV. It is concluded that the electron is transferred from $\beta$-phenylthiostyryl radical to thiophenol in a transition state. This indicates that stabilization of the transition state is induced by a significant contribution of structure 3 .

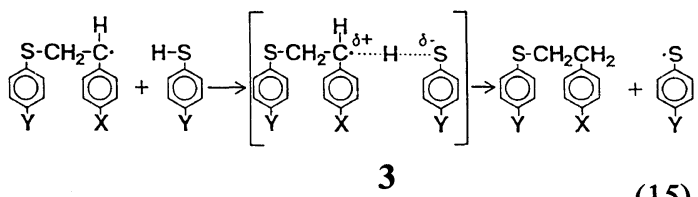

Better correlation with $\sigma^{+}$rather than $\sigma$ implies great contribution of the $\mathrm{S}-\mathrm{H}$ bond dissociation in the transition state. Similar idea was discussed in the study on the substituent effect of hydrogen abstraction from ring substituted toluene by Russel. ${ }^{23}$ Conseuqntly, it is realized that a perturbation between SOMO of intermediate carbon radical and LUMO of 
thiophenol in the transition state is an important controlling factor for this reaction; that is, the intermediate carbon radical is regarded as a nucleophilic one.

Ito et al. ${ }^{24}$ also determined the substituent effect on the addition reaction of substituted benzenethiyl radicals to styrene. In this case, the substituent effect did not obey eq 11 but eq 12 . The reaction constant was positive, $\rho=+1.37$.

Thus benzenethiyl radicals as electrophiles add to the vinyl group of styrenes, and $\beta$-phenylthiostyryl radicals as nucleophiles abstract hydrogen from thiophenols. That is, $\beta$-phenylthiostyryl radicals prefer thiophenols to styrenes and react hardly with styrenes. According to the reaction scheme of the model adduct formation, the authors propose the polyaddition mechanism. In the case of the polyaddition of BDT to DVB, two vinyl groups are exclusively assembled in the polymer backbone and there is no pendant vinyl group except polymer ends. That is, the polymer as well as the adducts have anti-Markownikoff's structures. The polymers obtained have soluble linear structures of high molecular weight $\left(\bar{M}_{w}=30000-1000000\right){ }^{7}$ Cross-linked polymer may be scarcely obtained in the polyaddition, because the polymer of the molecular weight of about 50,000 has potentially only one pendant vinyl group according to calculation based on experimental data obtained in the adduct preparation. The polyaddition of BDT to DVB can be regarded as the addition reaction of thiophenol having a thiol group to styrene having a vinyl group at the parapositions. However, $\sigma^{+}$for the $p$-vinyl and $p$-thiol groups are scarcely known. In the case of nitration of $\beta$-substituted styrene, $\sigma^{+}$for the $p$-vinyl group was determined as $-0.18^{25} . \sigma^{+}$ for the $p$-thiol substituent has been reported as $0.019,{ }^{26}$ based on rates of solvolysis of cumyl chlorides. The reactivity of the addition reaction of BDT to DVB estimated from Figures 7 (b) and 8 (b) using the above $\sigma^{+}$may be about nine times higher than that of the addition reaction of thiophenol to styrene. It was also found that the reactivity of the vinyl groups in DVB bis about five times higher than that of the vinyl group in $p$-(2-phenylthio)ethylstyrene $\left(\mathrm{CH}_{2}=\mathrm{CH} \phi \mathrm{CH}_{2} \mathrm{CH}_{2} \mathrm{~S} \phi\right)$ towards thiophenol. ${ }^{10}$ This suggests that the $p$-vinyl substituent increases the reactivity of styrene. Such a substituent effect of the para-positions may induce high polymerizability of DVB with BDT.

Acknowledgment. The authors are indebted to Mr. K. Tanaka (Idemitsu Kosan Co.) and Mr. M. Ozawa (Mitsubishi Petrochemical Co.) for the FD and GC mass spectra, respectively.

\section{REFERENCES}

1. T. Posner, Ber., 646 (1905).

2. F. Ashworth and G. N. Burkhadt, J. Chem. Soc., 1791 (1928); G. N. Burkhardt, Trans. Faraday Soc., 30, 18 (1934).

3. M. S. Kharasch and F. R. Mayo, J. Am. Chem. Soc., 55, 2468 (1934); S. O. Jones and E. E. Reid, ibid., 60, 2452 (1938).

4. M. S. Kharasch, A. T. Read, and F. R. Mayo, Chem . Ind., 57, 752 (1938).

5. M. Onyszchuk and C. Sivertz, Can. J. Chem., 33, 1034 (1955); R. H. Pallen and C. Sivertz, ibid., 35, 723 (1957).

6. C. S. Marvel and R. R. Chambers, J. Am. Chem. Soc., 70, 993 (1948); C. S. Marvel and P. H. Aldrich, ibid., 72, 1978 (1950); C. S. Marvel and H. W. Hill, Jr., ibid., 73, 481 (1951); C. S. Marvel and A. H. Markhart, Jr., ibid., 73, 1064 (1951); C. S. Marvel and A. Kotch, ibid., 73, 1100 (1951).

7. T. Yagi, G. Morishita, and E. Kobayashi, Zairyo Gijyutsu, 7(8), 16 (1989).

8. E. Kobayashi, T. Ohashi, and J. Furukawa, Makromol. Chem., 187, 2525 (1986); J. Polym. Sci., A, Polym. Chem., 25, 2077 (1987).

9. E. Kobayashi, J. Jiang, H. Matsumoto, and J. Furukawa, Polym. J., in press.

10. J. Jiang, E. Kobayashi, T. Obata, and J. Furukawa, Polym. J., in press.

11. R. Adains and C. R. Noller, Org. Synth., Coll. Vol. II, 109 (1955).

12. H. G. France, R. E. Lutz, and R. L. Wayland, J. Am. Chem. Soc., 72, 5511 (1950).

13. C. G. Overberger and H. H. Sounders, Org. Synth., Coll. Vol. III, 204 (1955). 
14. W. Schoeniger, Mikrochim. Acta, 123 (1955).

15. C. G. Screttas and M. Micha-Screttas, J. Org. Chem., 43, 1064 (1978).

16. M. Imoto, M. Kinoshita, and M. Nishizaki, Makromol. Chem., 86, 217 (1965).

17. H. C. Brown and Y. Okamoto, J. Am. Chem. Soc., 80, 4979 (1958).

18. T. Yamamoto and T. Otsu, J. Soc. Org. Chem. Jpn., 23, 643 (1965).

19. Y. Schaafsma, A. F. Bickel, and C. Kooyman, Rec. Trav. Chim., 76, 180 (1957).

20. M. Kinoshita and M. Imoto, Kougyo Kagaku Zasshi, 68(12), 2454 (1965).
21. T. Yamamoto, Polym. Lett., 4, 1039 (1966); Bull. Chem. Soc. Jpn., 40, 642 (1967); T. Yamamoto, T. Otsu, and M. Imoto, Kogyo Kagaku Zasshi, 69, 990 (1966); ibid., 70, 203 (1967).

22. M. Imoto, Kagaku, 29, 958 (1974).

23. G. A. Russell, J. Am. Chem. Soc., 79, 3871 (1957).

24. O. Ito and M. Matsuda, J. Am. Chem. Soc., 101(19), 5732 (1979).

25. W. F. Reynolds, T. A. Modro, E. Skorupowa, and A. Maron, Can. J. Chem., 58, 412 (1980).

26. C. G. Swain and E. C. Lupton, Jr., J. Am. Chem. Soc., 90, 4328 (1968). 